\title{
ON THE NOTION OF COEXISTENCE IN QUANTUM MECHANICS
}

\author{
Paul Busch* - JukKa Kiukas** - PeKKa Lahti*** \\ Dedicated to Professor Sylvia Pulmannová on the occasion of her 70th birthday \\ (Communicated by Anatolij Dvurečenskij)
}

\begin{abstract}
The notion of coexistence of quantum observables was introduced to describe the possibility of measuring two or more observables together. Here we survey the various different formalisations of this notion and their connections. We review examples illustrating the necessary degrees of unsharpness for two noncommuting observables to be jointly measurable (in one sense of the phrase). We demonstrate the possibility of measuring together (in another sense of the phrase) noncoexistent observables. This leads us to a reconsideration of the connection between joint measurability and noncommutativity of observables and of the statistical and individual aspects of quantum measurements.
\end{abstract}

(C) 2010

Mathematical Institute

Slovak Academy of Sciences

\section{Introduction}

The dual notions of states and observables are the basic ingredients for formulating the probability structure of quantum mechanics. If $\mathscr{H}$ is the (complex separable) Hilbert space associated with the quantum system, then the quantum mechanical (Born) probability formula is given by the trace formula $p_{\rho}^{E}(X)=\operatorname{tr}[\rho E(X)]$; here $\rho$ is the state of the quantum system, a positive trace one operator acting on $\mathscr{H}$, and $E: X \mapsto E(X)$ is the measured observable of the system, represented as a semispectral measure on a $\sigma$-algebra $\mathscr{A}$ of subsets of a set $\Omega$ with positive, unit bounded operators $E(X)$ acting on $\mathscr{H}$ as values. In this way any observable $E$ can be identified with the map $\rho \mapsto p_{\rho}^{E}$, that is, with the totality of its associated measurement outcome probability distributions.

2000 Mathematics Subject Classification: Primary 81P05, 81P15, 81P16.

Keywords: coexistent observables, joint measurability, noncommutativity, unsharpness. 


\section{PAUL BUSCH — JUKKA KIUKAS — PEKKA LAHTI}

The question of the possibility of measuring together (or jointly) two or more physical quantities lies at the heart of quantum mechanics. Insofar as the purpose of a measurement is to determine the probabilities for the various possible values of the measured observable, this question amounts to asking the following:

(Q) Given any two observables $\rho \mapsto p_{\rho}^{E_{1}}$ and $\rho \mapsto p_{\rho}^{E_{2}}$, with the value spaces $\left(\Omega_{1}, \mathscr{A}_{1}\right)$ and $\left(\Omega_{2}, \mathscr{A}_{2}\right)$, respectively, is there an observable $\rho \mapsto p_{\rho}^{E}$, with a value space $(\Omega, \mathscr{A})$, from which $E_{1}$ and $E_{2}$ can be reconstructed in an operationally feasible way?

There are three approaches which have been used extensively to analyse the question (Q). The first one has its origin in the theory of sequential measurements, the second refers directly to joint measurements, whereas the third arises from the functional calculus of observables. Question (Q) can be rephrased accordingly in three ways.

1) For which pairs of observables $E_{1}, E_{2}$ is the following statement true: for any $\rho$, there is a probability bimeasure

$$
\mathscr{A}_{1} \times \mathscr{A}_{2} \ni(X, Y) \mapsto p_{\rho}(X, Y) \in[0,1]
$$

such that $p_{\rho}^{E_{1}}$ and $p_{\rho}^{E_{2}}$ are its marginal measures, in the sense that $p_{\rho}\left(X, \Omega_{2}\right)=p_{\rho}^{E_{1}}(X)$ and $p_{\rho}\left(\Omega_{1}, Y\right)=p_{\rho}^{E_{2}}(Y)$ for any $X \in \mathscr{A}_{1}, Y \in \mathscr{A}_{2}$ ? If this is the case, $E_{1}$ and $E_{2}$ are said to have a biobservable, that is, there is a positive operator bimeasure $B: \mathscr{A}_{1} \times \mathscr{A}_{2} \rightarrow \mathscr{L}(\mathscr{H})$ such that $E_{1}(\cdot)=B\left(\cdot, \Omega_{2}\right)$ and $E_{2}(\cdot)=B\left(\Omega_{1}, \cdot\right)$.

2) For which pairs of observables $E_{1}, E_{2}$ is the following statement true: for any $\rho$, there is a joint probability measure ${ }^{1}$

$$
\mathscr{A}_{1} \otimes \mathscr{A}_{2} \ni Z \mapsto p_{\rho}(Z) \in[0,1]
$$

such that $p_{\rho}^{E_{1}}$ and $p_{\rho}^{E_{2}}$ are its marginal measures, that is, $p_{\rho}\left(X \times \Omega_{2}\right)=$ $p_{\rho}^{E_{1}}(X)$ and $p_{\rho}\left(\Omega_{1} \times Y\right)=p_{\rho}^{E_{2}}(Y)$ for any $X \in \mathscr{A}_{1}, Y \in \mathscr{A}_{2}$ ? If this is the case, then $E_{1}$ and $E_{2}$ are said to have a joint observable, that is, there is an observable $F: \mathscr{A}_{1} \otimes \mathscr{A}_{2} \rightarrow \mathscr{L}(\mathscr{H})$ such that $E_{1}(\cdot)=F\left(\cdot \times \Omega_{2}\right)$ and $E_{2}(\cdot)=F\left(\Omega_{1} \times \cdot\right)$.

3) For which pairs of observables $E_{1}, E_{2}$ is the following statement true: for any $\rho$, there is a probability measure $p_{\rho}$ defined on a $\sigma$-algebra $\mathscr{A}$ of a set $\Omega$ and measurable functions $f_{1}: \Omega \rightarrow \Omega_{1}$ and $f_{2}: \Omega \rightarrow \Omega_{2}$ such that $p_{\rho}\left(f_{1}^{-1}(X)\right)=p_{\rho}^{E_{1}}(X)$ and $p_{\rho}\left(f_{2}^{-1}(Y)\right)=p_{\rho}^{E_{2}}(Y)$ for any $X \in \mathscr{A}_{1}$, $Y \in \mathscr{A}_{2}$ ? If this is the case, $E_{1}, E_{2}$ are said to be functions of $E$, in the sense that $E_{1}=E \circ f_{1}^{-1}$ and $E_{2}=E \circ f_{2}^{-1}$.

\footnotetext{
$1_{\mathscr{A}_{1}} \otimes \mathscr{A}_{2}$ denotes the $\sigma$-algebra of subsets of $\Omega_{1} \times \Omega_{2}$ generated by the sets $X \times Y, X \in \mathscr{A}_{1}$,
} $Y \in \mathscr{A}_{2}$. 


\section{COEXISTENCE IN QUANTUM MECHANICS}

If $E_{1}$ and $E_{2}$ have a joint observable, then they also are functions of an observable, and if they are functions of an observable, then they have a biobservable. In general, a biobservable is not induced by a joint observable. However, if the measurable spaces involved are sufficiently regular, then such pathologies do not exist. Indeed, if the value spaces $\left(\Omega_{1}, \mathscr{A}_{1}\right),\left(\Omega_{2}, \mathscr{A}_{2}\right),(\Omega, \mathscr{A})$ are Borel spaces, that is, the sets are locally compact metrizable and separable topological spaces and the $\sigma$-algebras are the Borel $\sigma$-algebras, ${ }^{2}$ then the three conditions are equivalent [2], see also [23, 24].

Example 1.1. As a first illustration, consider any two observables $E_{1}: \mathscr{A}_{1} \rightarrow \mathscr{L}(\mathscr{H})$ and $E_{2}: \mathscr{A}_{2} \rightarrow \mathscr{L}(\mathscr{H})$. If they commute with each other, that is, $E_{1}(X) E_{2}(Y)=E_{2}(Y) E_{1}(X)$, for all $X \in \mathscr{A}_{1}, Y \in \mathscr{A}_{2}$, then the map $(X, Y) \mapsto E_{1}(X) E_{2}(Y)$ is a biobservable for $E_{1}$ and $E_{2}$. If the value spaces are Borel spaces, then $E_{1}$ and $E_{2}$ have a joint observable $F$ with the property $F(X \times Y)=E_{1}(X) E_{2}(Y)$. If, in addition, one of the observables is projection valued, then $F$ is the unique joint observable of $E_{1}$ and $E_{2}$. This follows directly from the fact that in such a case $E_{1}(X) E_{2}(Y)$ is the greatest lower bound of the effects $E_{1}(X)$ and $E_{2}(Y)$ ([29]), for a slightly different argument, see, e.g. [15].

Though important, the above three reformulations of question (Q) do not exhaust its content. Below we shall describe yet another way of phrasing and answering this question. Further, we will give examples of jointly measurable pairs of (generally noncommuting) observables and review some necessary and sufficient conditions for their joint measurability. This will enable us to identify significant differences between the various notions of joint measurability considered here.

We start with a brief description of the notion of coexistence of observables, which has been introduced as a seemingly obvious generalization of the idea of a joint observable for a pair of observables with finitely many values, and which encompasses the three notions of joint measurability arising from the above formalizations of $(\mathrm{Q})$.

\section{Coexistence}

Observables $E_{1}: \mathscr{A}_{1} \rightarrow \mathscr{L}(\mathscr{H})$ and $E_{2}: \mathscr{A}_{2} \rightarrow \mathscr{L}(\mathscr{H})$ are coexistent if there is an observable $E: \mathscr{A} \rightarrow \mathscr{L}(\mathscr{H})$ such that

$$
\left\{E_{1}(X): X \in \mathscr{A}_{1}\right\} \cup\left\{E_{2}(Y): Y \in \mathscr{A}_{2}\right\} \subseteq\{E(Z): Z \in \mathscr{A}\} .
$$

Such an observable $E$ will be called an encompassing observable for $E_{1}$ and $E_{2}$. Clearly, if $E_{1}: \mathscr{A}_{1} \rightarrow \mathscr{L}(\mathscr{H})$ and $E_{2}: \mathscr{A}_{2} \rightarrow \mathscr{L}(\mathscr{H})$ have a joint observable

\footnotetext{
${ }^{2}$ Then also $\mathscr{B}\left(\Omega_{1}\right) \otimes \mathscr{B}\left(\Omega_{2}\right)=\mathscr{B}\left(\Omega_{1} \times \Omega_{2}\right)$.
} 


\section{PAUL BUSCH — JUKKA KIUKAS — PEKKA LAHTI}

or if they are functions of an observable, then $E_{1}$ and $E_{2}$ are also coexistent. Moreover, if the value spaces involved are Borel spaces, then $E_{1}$ and $E_{2}$ are coexistent whenever they have a biobservable. In spite of many attempts ([21, $23,22,13])$ the question has remained open whether the notion of coexistence is actually more general than these other three (essentially equivalent) notions of joint measurability.

Let $(\Omega, \mathscr{A})$ and $\left(\Omega_{1}, \mathscr{A}_{1}\right)$ be any two measurable spaces. Then for any observable $E: \mathscr{A} \rightarrow \mathscr{L}(\mathscr{H})$ and measurable function $f: \Omega \rightarrow \Omega_{1}$, the range $\operatorname{ran}\left(E^{f}\right)$ of the image observable $E^{f}: X \mapsto E^{f}(X)=E\left(f^{-1}(X)\right)$ is contained in the range of $E$. The main problem is in the converse implication, that is: if $E_{1}: \mathscr{A}_{1} \rightarrow \mathscr{L}(\mathscr{H})$ is an observable with the property $\operatorname{ran}\left(E_{1}\right) \subseteq \operatorname{ran}(E)$, can one construct a function $f: \Omega \rightarrow \Omega_{1}$ such that $E_{1}=E^{f} ?^{3}$ The classic results of Sikorski [32] and Varadarajan [36], see also [30] and [13], show that such a construction is possible if the ranges are separable Boolean algebras. Example 2.2 below is an application of this result. Yet the Boolean nature of the ranges of observables is not necessary for their functional calculus; some physically relevant examples have been studied in [14]. Before recalling the Boolean case we shall note another example where the above problem is resolved, namely the case where one of the observables is projection valued.

Example 2.1. If $E_{1}$ and $E_{2}$ are coexistent, and if one of them is projection valued, then $E_{1}$ and $E_{2}$ commute with each other [26, Th. 1.3.1, p. 91], so that the map $(X, Y) \mapsto E_{1}(X) E_{2}(Y)$ is a biobservable of $E_{1}$ and $E_{2}$. If, in addition, the value spaces are Borel spaces, then they have a joint observable $F$, which, by Example 1.1 is necessarily of the product form $F(X \times Y)=E_{1}(X) E_{2}(Y)$.

Let $\mathscr{E}(\mathscr{H})=\{A \in \mathscr{L}(\mathscr{H}): O \leq A \leq I\}$ be the set of effect operators. $\mathscr{E}(\mathscr{H})$ is equipped with the partial order $\leq$ (of selfadjoint operators) and the complementation map $A \mapsto A^{\perp}:=I-A$. For an observable $E: \mathscr{A} \rightarrow \mathscr{E}(\mathscr{H})$, the range $\operatorname{ran}(E)=\{E(X): X \in \mathscr{A}\}$ is not, in general, a Boolean sub- $\sigma$-algebra of $\mathscr{E}(\mathscr{H})$, that is, the map $\mathscr{A} \ni X \mapsto E(X) \in \mathscr{E}(\mathscr{H})$ is not necessarily a $\sigma$-homomorphism, notwithstanding the fact that $\mathscr{A}$ is a Boolean $\sigma$-algebra (of subsets of $\Omega$ ). It is an easy exercise to check that $\operatorname{ran}(E)$ is a Boolean subsystem of $\mathscr{E}(\mathscr{H})$ if an only if $E$ is projection valued. It may, however, happen that $E$ is a $\sigma$-homomorphism from $\mathscr{A}$ to $(\operatorname{ran}(E), \leq, \perp)$ without $E$ being projection valued. Indeed, for a given $E$ the system $(\operatorname{ran}(E), \leq, \perp)$ is Boolean if and only if $E$ is regular $([21,13])$. We recall that $E$ is regular if there is no nontrivial effect operator $E(X)(\neq O, I)$ of $\operatorname{ran}(E)$ which would be either below $\frac{1}{2} I$ or above $\frac{1}{2} I$.

Example 2.2. If $E_{1}$ and $E_{2}$ are coexistent with an encompassing observable $E$ that is regular, then $\operatorname{ran}\left(E_{1}\right)$ and $\operatorname{ran}\left(E_{2}\right)$ are Boolean sub- $\sigma$-algebras of $\operatorname{ran}(E)$.

\footnotetext{
${ }^{3}$ The condition $\operatorname{ran}\left(E_{1}\right) \subseteq \operatorname{ran}(E)$ need not imply that $E_{1}$ is a function of $E$; for an example, see [13, Remark 1.1].
} 


\section{COEXISTENCE IN QUANTUM MECHANICS}

If the value spaces involved are complete separable metric spaces with the cardinality of $\mathbb{R}$, then $E_{1}$ and $E_{2}$ are functions of $E$. In particular, if the value spaces are real Borel spaces $(\mathbb{R}, \mathscr{B}(\mathbb{R}))$, then the regularity of an encompassing observable $E$ implies the existence of a biobservable $B$, a joint observable $F$, and Borel functions $f_{1}$ and $f_{2}$, such that $B(X, Y)=F(X \times Y)=E\left(f_{1}^{-1}(X) \cap f_{2}^{-1}(Y)\right)$ for all $X, Y \in \mathscr{B}(\mathbb{R})$, see e.g. $[21,13]$.

The problem with the notion of coexistence is that in itself, it does not entail a constructive procedure for identifying an encompassing observable $E$ for $E_{1}$, $E_{2}$, nor for the embedding of the ranges of the latter into the former. If it is given that observables $E_{1}$ and $E_{2}$ are coexistent with encompassing observable $E$, then all that is known is that there exists, for each $X \in \mathscr{A}_{1}$, a set $Z_{X} \in \mathscr{A}$ such that $E_{1}(X)=E\left(Z_{X}\right)$, and similarly for $E_{2}$. On the basis of this information only, there seems to be no way to pick out the effect operators of $\operatorname{ran}\left(E_{1}\right)$ from those of $\operatorname{ran}(E)$, and similarly for $E_{2}$. Therefore, there seems to be no operational way to use the statistics $\rho \mapsto p_{\rho}^{E}$ to reconstruct the statistics of $E_{1}$ or $E_{2}$.

By contrast, the notion of joint measurability does provide such a procedure and is, in addition, naturally adapted to the quantum mechanical modeling of measurement processes as we will recall next.

\section{Measurement theory}

According to the quantum theory of measurement, any observable (as a semispectral measure) $E: \mathscr{A} \rightarrow \mathscr{L}(\mathscr{H})$ admits a measurement dilation of the form

$$
E(X)=V_{\phi}^{*} U^{*} I \otimes P(X) U V_{\phi}
$$

where $U: \mathscr{H} \otimes \mathscr{K} \rightarrow \mathscr{H} \otimes \mathscr{K}$ is a unitary operator modeling the measurement coupling between the measured system (with the Hilbert space $\mathscr{H}$ ) and the apparatus (or the probe system, with the Hilbert space $\mathscr{K}$ ), $V_{\phi}$ is the embedding $\mathscr{H} \rightarrow \mathscr{H} \otimes \mathscr{K}, \varphi \mapsto \varphi \otimes \phi$, with $\phi$ being the initial probe (vector) state, and $P: \mathscr{A} \rightarrow \mathscr{L}(\mathscr{K})$ is the probe observable (which can be taken to be a spectral measure). We let $\mathscr{M}=(\mathscr{K}, P, U, \phi)$ denote the measurement realization (1) of the observable $E$.

Let $\left(\Omega_{1}, \mathscr{A}_{1}\right)$ be any other measurable space, and let $f: \Omega \rightarrow \Omega_{1}$ be a measurable function, called a pointer function. The pointer function $f$ and the measurement $\mathscr{M}$ define another observable $E_{1}$, obtained as the image of $E$ un$\operatorname{der} f$,

$$
E_{1}(X)=E\left(f^{-1}(X)\right), \quad X \in \mathscr{A}_{1} .
$$

Clearly, $\operatorname{ran}\left(E_{1}\right) \subseteq \operatorname{ran}(E)$, and, although $\mathscr{M}$ is not an $E_{1}$-measurement, the measurement $\mathscr{M}$ together with the pointer function $f$ constitutes a measurement of $E_{1}$. In particular, if any two observables $E_{1}$ and $E_{2}$ are functions of a third 
observable $E$, then any $E$-measurement $\mathscr{M}$ serves also as a measurement of both $E_{1}$ and $E_{2}$.

It may occur that one can use the measurement statistics to construct the statistics of another observable without using such a functional calculus. We describe next such a possibility.

\section{The method of moments}

We now review a possibility of determining the statistics of an observable from the statistics of another observable without the use of a functional calculus.

The method of moments refers to a case where from the moments of the actually measured statistics one is able to infer the moments, and eventually the whole statistics of another observable. Typically, such a situation arises when the actually performed measurement constitutes an unsharp measurement of another obervables.

To describe this method in more detail, let $E_{\varphi, \psi}$ denote the complex measure $Y \mapsto E_{\varphi, \psi}(Y)=\langle\varphi \mid E(Y) \psi\rangle$ defined by an observable $E: \mathscr{B}(\mathbb{R}) \rightarrow L(\mathscr{H})$ and the vectors $\varphi, \psi \in \mathscr{H}$. In particular, if $\varphi \in \mathscr{H}$ is a unit vector, then $E_{\varphi, \varphi}=p_{\rho}^{E}$, with $\rho=|\varphi\rangle\langle\varphi|$. We recall that the $k$ th moment operator $E[k]$ of $E$ is the weakly defined operator $E[k]=\int_{\mathbb{R}} x^{k} \mathrm{~d} E(x)$, with the domain $\mathscr{D}(E[k])$ consisting of those vectors $\psi \in \mathscr{H}$ for which the integral $\int x \mathrm{~d} E_{\varphi, \psi}(x)$ exists for all $\varphi \in \mathscr{H}$. In particular, if the integrals $\int_{\mathbb{R}} x^{k} \mathrm{~d} p_{\rho}^{E}(x)$ exist, they define the moments of the measurement outcome statistics $p_{\rho}^{E}$.

Let $\mu: \mathscr{B}(\mathbb{R}) \rightarrow[0,1]$ be a probability measure, and let $\mu * E$ denote the convolution of $\mu$ and $E$. It is the observable $X \mapsto(\mu * E)(X)$ defined by $\langle\varphi \mid(\mu * E)(X) \psi\rangle=\mu * E_{\varphi, \psi}(X), \varphi, \psi \in \mathscr{H}$, where $\mu * E_{\varphi, \psi}$ is the convolution of $\mu$ with the complex measure $E_{\varphi, \psi}(Y)$, that is,

$$
\mu * E_{\varphi, \psi}(X)=\int_{\mathbb{R}} \mu(X-y) \mathrm{d} E_{\varphi, \psi}(y) .
$$

We note that $\operatorname{ran}(E)$ is contained in $\operatorname{ran}(\mu * E)$ only if $\mu$ is a point measure. However, it may happen that one can reconstruct (the moments of) $E$ from (the moments of) $\mu * E$ in such a way that the full statistics become uniquely determined. Indeed, the moment operators of $\mu * E$ and $E$ are related with each other as follows ([19]). 
LEMMA 4.1. Let $E: \mathscr{B}(\mathbb{R}) \rightarrow L(\mathscr{H})$ be a semispectral measure, and $\mu: \mathscr{B}(\mathbb{R}) \rightarrow$ $[0,1]$ a probability measure. If $\mu[k]$ exists, then $\mathscr{D}(E[k]) \subset \mathscr{D}((\mu * E)[k])$, and

$$
(\mu * E)[k] \supset \sum_{n=0}^{k}\left(\begin{array}{l}
k \\
n
\end{array}\right) \mu[k-n] E[n] .
$$

Assume now that all the moments $\mu[k]$ of the blurring probability measure $\mu$ are finite and in addition that $\emptyset \neq D \subset \bigcap_{k=0}^{\infty} \mathscr{D}(E[k])$. Then for any state $\rho=|\varphi\rangle\langle\varphi|, \varphi \in D$

$$
p_{\rho}^{\mu * E}[k]=\sum_{n=0}^{k}\left(\begin{array}{l}
k \\
n
\end{array}\right) \mu[k-n] p_{\rho}^{E}[n],
$$

from which a recursion formula for the moments $p_{\rho}^{E}[k]$ is obtained:

$$
p_{\rho}^{E}[k]=p_{\rho}^{\mu * E}[k]-\sum_{n=0}^{k-1}\left(\begin{array}{l}
k \\
n
\end{array}\right) \mu[k-n] p_{\rho}^{E}[n] .
$$

Assume, further, that the moments $p_{\rho}^{E}[k]$ fulfill the operationally verifiable condition

$$
\left|p_{\rho}^{E}[k]\right| \leq C R^{k} k !, \quad k=1,2, \ldots
$$

This implies that $\int \mathrm{e}^{a|x|} \mathrm{d} p_{\rho}^{E}<\infty$, whenever $0<a<1 / 2 R$ (see, e.g. [33, Proposition 1.5]), showing that the probability measure $p_{\rho}^{E}$ is exponentially bounded, a condition which assures that the moment sequence $\left(p_{\rho}^{E}[k]\right)_{k=0}^{\infty}$ uniquely determines the probability measure $p_{\rho}^{E}$, see, e.g., [3, p. 406, Theorem 30.1]. If the set $D$ above is a dense subspace, then the probability measures $p_{\rho}^{E}, \rho=|\varphi\rangle\langle\varphi|$, $\varphi \in D$, determine, by the polarization identity, the observable $E$. Note that if $E$ is a spectral measure, then there always exists such a dense subspace $D$ so that it only remains to check that the convolving measure $\mu$ has finite moments and that the condition (7) is satisfied for a sufficiently large set of states $\rho$.

We conclude that under the operational conditions specified above one can reconstruct the moments of $p_{\rho}^{E}$ and then uniquely determine the corresponding statistics, from the actually measured distribution $p_{\rho}^{\mu * E}$ using the method of moments even though the range of $E$ may not be contained in the range of $\mu * E$. One may call this an indirect $E$-measurement. 


\section{Examples: \\ Measuring together sharp noncommuting observables}

\subsection{Indirect measurement of sharp position and momentum}

Let $\mathrm{Q}$ and $\mathrm{P}$ denote the spectral measures of the position and momentum operators $Q$ and $P$, acting in $L^{2}(\mathbb{R})$. The convolutions $\mu * \mathrm{Q}$ and $\nu * \mathrm{P}$ of $\mathrm{Q}$ and $\mathrm{P}$ with probability measures $\mu, \nu: \mathscr{B}(\mathbb{R}) \rightarrow[0,1]$ are unsharp position and momentum observables, respectively. The standard (von Neumann) model of a position measurement constitutes a realization of $\mu * \mathrm{Q}$ where $\mu$ is an absolutely continuous probability measure depending on the preparation of the measurement probe and the coupling strength between probe and particle (for details, see $[8])$.

Consider a measurement of $\mu * \mathrm{Q}$. Assuming that all the moments of $\mu$ are finite, for instance, in the standard model the initial probe state is a (compactly supported) slit-state or a Gaussian state, and choosing $D$ to be, for instance, the linear span of the (normalized) Hermite functions, then the moments $p_{\rho}^{\mathrm{Q}}[k]$, $k \in \mathbb{N}$, can be obtained recursively by (6) from the actually measured statistics $p_{\rho}^{\mu * \mathrm{Q}}, \rho=|\varphi\rangle\langle\varphi|, \varphi \in D$, and they fulfill the condition (7). Therefore, the numbers $p_{\rho}^{\mathrm{Q}}[k], k \in \mathbb{N}$, determine the distribution $p_{\rho}^{\mathrm{Q}}$. Due to the density of $D$, the actual measurement $\rho \mapsto p_{\rho}^{\mu * \mathrm{Q}}$ thus determines the whole observable $\rho \mapsto p_{\rho}^{\mathrm{Q}}$, that is, the (sharp) position observable $\mathrm{Q}$ is measured indirectly by a measurement of an unsharp position $\mu * \mathrm{Q}$, whenever all moments of the blurring measure $\mu$ are finite.

Similarly, an unsharp momentum measurement can serve as an indirect measurement of the sharp momentum.

Sharp position and momentum observables $Q$ and $P$ are (strongly) noncommutative. Therefore, they are noncoexistent, they do not have a biobservable or joint observable, nor are they functions of a third observable. Nevertheless they can be measured together indirectly, that is, there are measurements $\mathscr{M}$ which allow one to determine, from the actual statistics $\rho \mapsto p_{\rho}^{E}$, both the position statistics $\rho \mapsto p_{\rho}^{\mathrm{Q}}$ and the momentum statistics $\rho \mapsto p_{\rho}^{\mathrm{P}}$.

The Weyl operators representing phase space translations by a displacement $(q, p) \in \mathbb{R}^{2}$ are defined as $W_{q p}=\mathrm{e}^{\mathrm{i} \frac{1}{2} q p} \mathrm{e}^{-\mathrm{i} q P} \mathrm{e}^{\mathrm{i} p Q}$. It is well known that any covariant phase space observable $G^{T}$ is generated by a positive operator $T$ of trace one (acting in $L^{2}(\mathbb{R})$ ) such that for $Z \in \mathscr{B}\left(\mathbb{R}^{2}\right)$,

$$
G^{T}(Z)=\frac{1}{2 \pi} \int_{Z} W_{q p} T W_{q p}^{*} \mathrm{~d} q \mathrm{~d} p .
$$

The Cartesian marginal observables of $G^{T}$ are the unsharp position and momentum observables $\mu * \mathrm{Q}$ and $\nu * \mathrm{P}$, with $\mu$ and $\nu$ defined by the Fourier related 


\section{COEXISTENCE IN QUANTUM MECHANICS}

densities $f(q)=\sum_{i} t_{i}\left|\eta_{i}(-q)\right|^{2}$ and $g(p)=\sum_{i} t_{i}\left|\hat{\eta}_{i}(-p)\right|^{2}$, where $T=\sum_{i} t_{i}\left|\eta_{i}\right\rangle\left\langle\eta_{i}\right|$ is the spectral decomposition of the generating operator $T$. Choosing the generating operator $T$ such that all the moments $\mu[k]$ and $\nu[k], k \in \mathbb{N}$, are finite, and using $D$ as given above, we conlude that the marginal measurement statistics $\rho \mapsto p_{\rho}^{\mu * \mathrm{Q}}$ and $\rho \mapsto p_{\rho}^{\nu * \mathrm{P}}, \rho=|\varphi\rangle\langle\varphi|, \varphi \in D$, collected under a single measurement scheme, suffice to determine both the position statistics $\rho \mapsto p_{\rho}^{\mathrm{Q}}$ and the momentum statistics $\rho \mapsto p_{\rho}^{\mathrm{P}}$.

The Arthurs-Kelly model, or a sequential standard position measurement followed by a sharp momentum measurement, or the eight-port homodyne detection scheme provide examples of measurement realizations of such a joint determination of the position and momentum statistics. For a more detailed discussion of these models, see, for example, [4, 31, 35, 8, 16, 10, 18].

\subsection{Indirect measurement of spin- $\frac{1}{2}$ components}

For observables with discrete or even finite sets of outcomes it becomes particularly simple to consider the question of indirect measurements. Let $P_{1}, P_{2}, \ldots, P_{n}$ be a complete family of mutually orthogonal projections such that $\sum_{k} P_{k}=I$, and let $\left(\lambda_{j k}\right)$ be a stochastic $m \times n$ matrix, that is, $\lambda_{j k} \geq 0, \sum_{k} \lambda_{k}=1$. Then the operators $E_{j}=\sum_{k} \lambda_{j k} P_{k}$ are positive and satisfy $\sum_{j} E_{j}=I$, that is, they constitute an observable which is a smeared version of the sharp observable defined by the $P_{k}$. If the matrix $\left(\lambda_{j k}\right)$ is square and invertible, it follows that the $P_{k}$ can be expressed as linear combinations of the $E_{j}$, so that $P_{k}=\sum_{j} \mu_{k j} E_{j}$. It follows that $\operatorname{tr}\left[\rho P_{k}\right]=\operatorname{tr}\left[\rho^{\prime} P_{k}\right]$ for all $k$ if and only if $\operatorname{tr}\left[\rho E_{j}\right]=\operatorname{tr}\left[\rho^{\prime} E_{j}\right]$; in other words, the observables given by $\left\{P_{k}: k=1,2, \ldots, n\right\}$ and $\left\{E_{j}: j=1,2, \ldots, n\right\}$ are equally good at separating distinct states, they are informationally equivalent.

As an example, we consider the joint determination of the statistics of noncommuting spin components of a spin- $\frac{1}{2}$ system. Using the bijective correspondence between $M\left(\mathbb{C}^{2}\right)$ and $\mathbb{C}^{4}$ mediated by the Pauli basis $I, \sigma_{1}, \sigma_{2}, \sigma_{3}$, we recall that any operator $(2 \times 2$ matrix $)$ can be written as $A=a_{0} I+\mathbf{a} \cdot \boldsymbol{\sigma}$. A state $\rho$ is given by $\rho=\frac{1}{2}(I+\mathbf{r} \cdot \boldsymbol{\sigma})$, with $\mathbf{r} \in \mathbb{R}^{3},|\mathbf{r}| \leq 1$. The following four-outcome observable $G$ is an example of a joint observable for smeared versions of the sharp observables $\sigma_{1}$ and $\sigma_{2}$ :

$$
\{+,-\} \times\{+,-\} \ni(j, k) \mapsto G_{j k}=\frac{1}{4}\left(I+\mathbf{n}_{j k} \cdot \boldsymbol{\sigma}\right),
$$


where $\mathbf{n}_{+, \pm}=\left(\mathbf{e}_{1} \pm \mathbf{e}_{2}\right) / \sqrt{2}, \mathbf{n}_{-, \pm}=\left(-\mathbf{e}_{1} \pm \mathbf{e}_{2}\right) / \sqrt{2}$. The two obvious marginal observables are given by the following pairs of effects:

$$
\begin{aligned}
E_{ \pm}^{(1)} & =G_{ \pm,+}+G_{ \pm,-}=\frac{1}{2}\left(I \pm \frac{\sqrt{2}}{2} \sigma_{1}\right) \\
& =\frac{1}{2}\left(1 \pm \frac{\sqrt{2}}{2}\right) P_{+}^{(1)}+\frac{1}{2}\left(1 \mp \frac{\sqrt{2}}{2}\right) P_{-}^{(1)}, \\
E_{ \pm}^{(2)} & =G_{+, \pm}+G_{-, \pm}=\frac{1}{2}\left(I \pm \frac{\sqrt{2}}{2} \sigma_{2}\right) \\
& =\frac{1}{2}\left(1 \pm \frac{\sqrt{2}}{2}\right) P_{+}^{(2)}+\frac{1}{2}\left(1 \mp \frac{\sqrt{2}}{2}\right) P_{-}^{(2)} .
\end{aligned}
$$

Here the $P_{ \pm}^{(k)}:=\frac{1}{2}\left(I \pm \sigma_{k}\right)$ are the spectral projections of $\sigma_{k}$. It is obvious that the transformations $\left\{P_{ \pm}^{(k)}\right\} \rightarrow\left\{E_{ \pm}^{(k)}\right\}$ are invertible. In fact, we have:

$$
P_{ \pm}^{(k)}= \pm \frac{1}{2}(\sqrt{2} \pm 1) E_{+}^{(k)} \mp \frac{1}{2}(\sqrt{2} \mp 1) E_{-}^{(k)} .
$$

Thus the statistics $p_{\rho}^{\sigma_{k}}, k=1,2$, can be reconstructed from the statistics of $E^{(k)}$ via measuring the joint observable $G$.

Realistic models of the measurement of a observable of the form $\left\{G_{j k}\right\}$ were first presented in [6]. A systematic study of the reconstruction of sharp spin$\frac{1}{2}$ observables from such non-ideal or approximate joint measurements can be found in [27].

\section{The method of state reconstruction}

The state $\rho$ of a quantum particle is not determined by its position and momentum distributions $p_{\rho}^{\mathrm{Q}}$ and $p_{\rho}^{\mathrm{P}}$. This is a well-known but important nonclassical feature of the quantum theory, a feature called surplus information by C. F. von Weizsäcker [37].

If an observable $E$ is informationally complete, that is, the map $\rho \mapsto p_{\rho}^{E}$ separates states, and if one can determine an algorithm for reconstructing the state $\rho$ from the statistics $p_{\rho}^{E}$, then one can obtain the measurement statistics of any observable, in particular the statistics $p_{\rho}^{\mathrm{Q}}$ and $p_{\rho}^{\mathrm{P}}$. Feasible state reconstruction algorithms are known for quadrature distributions, ${ }^{4}$ and simple phase space observables (see [25], and also [17]). Hence, it is clear that the question (Q) can have a positive answer without the observables being coexistent.

The reconstruction scheme of Subsection 5.1 shows, however, that there are single measurement schemes which allow one to reconstruct, in an operational way, the moments of the distributions $p_{\rho}^{\mathrm{Q}}$ and $p_{\rho}^{\mathrm{P}}$ without the need to reconstruct first the state $\rho$; in fact, in the example of a phase space observable $G^{T}$, it is

\footnotetext{
${ }^{4}$ Note that these can easily be bunched together to form a single informationally complete observable.
} 
not necessary to require that $G^{T}$ is informationally complete. ${ }^{5}$ For a suitable subclass of states, the moments contain the same information as the distributions themselves, so one may speak of a measurement. (Obviously, a serious disadvantage of this method compared to the state reconstruction is that the distributions cannot be algorithmically reconstructed from the moments.)

In the case of a spin- $\frac{1}{2}$ system, it is possible to obtain simultaneous reconstructions of the spin component observables $\sigma_{\mathbf{n}}$ along all directions (specified by the unit vectors $\mathbf{n}$ ) from a single observable $M$ as follows. Let $\left(S^{2}, \mathscr{B}\left(S^{2}\right)\right)$ denote the unit 2 -sphere in $\mathbb{R}^{3}$, equipped with the standard Borel $\sigma$-algebra, and let $\mathrm{d} \Omega(\mathbf{n})$ be the uniform surface measure normalised as $\Omega\left(S^{2}\right)=4 \pi$. The following is a normalized positive operator measure:

$$
\mathscr{B}\left(S^{2}\right) \ni Z \mapsto M(Z):=\frac{1}{2 \pi} \int_{Z} \frac{1}{2}(I+\mathbf{n} \cdot \boldsymbol{\sigma}) \mathrm{d} \Omega(\mathbf{n}) .
$$

It is obvious that one can define, for any direction, a 2-valued marginal observable by choosing a partition of $S^{2}$ in the form of two complementary hemispheres with poles along the given direction. For simplicity, we consider the direction along the $z$-axis of some Cartesian coordinate system, and denote the partition as $Z_{ \pm}$. Then we obtain:

$$
M\left(Z_{ \pm}\right) \equiv M_{ \pm}=\frac{1}{2}\left(I \pm \frac{1}{2} \sigma_{3}\right) .
$$

As before, the statistics of $\sigma_{3}$ can be reconstructed from this marginal observable.

\section{Joint measurability of noncommuting observables}

The examples of the preceding sections illustrate the following well-known fundamental fact. Noncommuting sharp observables do not admit any joint observable; but there are smeared versions of such noncommuting observables that do possess joint observables. The natural question about the necessary amount of unsharpness required to ensure joint measurability has not yet been answered in full generality, but important insights have been gained in special cases.

In the case of position and momentum it is known $[38,12]$ that observables $\mu * \mathrm{Q}$ and $\nu * \mathrm{P}$ are jointly measurable if and only if the smearing measures $\mu$, $\nu$ have Fourier-related densities, in the sense described in Subsection 5.1. In this case the variances of these measures, which represent measures of the inaccuracies of the position and momentum determination, satisfy the Heisenberg

${ }^{5}$ The informational completeness of $G^{T}$ is known to be equivalent to the assumption that $\operatorname{tr}\left[W_{q p} T\right] \neq 0$ for almost all $(q, p) \in \mathbb{R}^{2}([1,20])$. 
uncertainty relation,

$$
\operatorname{Var}(\mu) \operatorname{Var}(\nu) \geq \frac{\hbar^{2}}{4}
$$

which thus is seen to constitute a necessary condition of joint measurability.

In the case of a spin- $\frac{1}{2}$ system or, more generally, a qubit represented by a 2 -dimensional Hilbert space $\mathbb{C}^{2}$, the question of necessary and sufficient conditions for the coexistence (equivalently, joint measurability) of a pair of effects has recently been completely answered $([11,34,39])$. Effects $A=a_{0} I+\mathbf{a} \cdot \boldsymbol{\sigma}$ and $B=b_{0} I+\mathbf{b} \cdot \boldsymbol{\sigma}$ are coexistent if and only if a certain inequality holds which can be cast in the following form [11]:

$$
\frac{1}{2}[\mathscr{F}(2-\mathscr{B})+\mathscr{B}(2-\mathscr{F})]+(x y-4 \mathbf{a} \cdot \mathbf{b})^{2} \geq 1 .
$$

Here the following abbreviations are used:

$$
\begin{aligned}
\mathscr{F} & :=\varphi(A)^{2}+\varphi(B)^{2} \\
\mathscr{B} & :=\beta(A)^{2}+\beta(B)^{2} \\
x & :=\varphi(A) \beta(A)=2 a_{0}-1 \\
y & :=\varphi(B) \beta(B)=2 b_{0}-1 ; \\
\varphi(A) & :=\sqrt{a_{0}^{2}-|\mathbf{a}|^{2}}+\sqrt{\left(1-a_{0}\right)^{2}-|\mathbf{a}|^{2}} \\
\beta(A) & :=\sqrt{a_{0}^{2}-|\mathbf{a}|^{2}}-\sqrt{\left(1-a_{0}\right)^{2}-|\mathbf{a}|^{2}} .
\end{aligned}
$$

$\varphi(B)$ and $\beta(A)$ are defined similarly. The quantity $\varphi(A)$ is a measure of the degree of unsharpness of the effect $A$, and $\beta(A)$ and $x$ are measures of the bias of $A$. An effect $A$ (and its complement $A^{\prime}=I-A$ ) is unbiased if the mid-point of its spectrum is $\frac{1}{2}$. (A more detailed investigation of these properties and measures can be found in [7].) The degree of noncommutativity is represented by the deviation of the term $|\mathbf{a} \cdot \mathbf{b}|$ from $\|\mathbf{a}\|\|\mathbf{b}\|$. This inequality represents a rather complicated trade-off between the unsharpness, bias and noncommutativity degrees of the two effects $A, B$, which must hold if they are to be jointly measurable.

In the special case of unbiased effects $\left(a_{0}=b_{0}=\frac{1}{2}\right)$, the above coexistence inequality assumes the simple form

$$
16|\mathbf{a} \times \mathbf{b}|^{2} \leq\left(1-4|\mathbf{a}|^{2}\right)\left(1-4|\mathbf{b}|^{2}\right)
$$

Considering that $A=\frac{1}{2} I+\mathbf{a} \cdot \boldsymbol{\sigma}$ is a projection if and only if $|\mathbf{a}|=\frac{1}{2}$, we see that $1-4|\mathbf{a}|^{2}$ is a measure of the unsharpness of $A$. Hence the product of the degrees of unsharpness of $A, B$ is bounded below by the square of the vector product of $\mathbf{a}$ and $\mathbf{b}$, which is proportional to the commutator of $A$ and $B$. 


\section{COEXISTENCE IN QUANTUM MECHANICS}

\section{Discussion}

We have reviewed three notions of joint measurability (based on biobservables, joint observables and functional calculus, respectively), the notion of coexistence, and a concept of indirect measurement, and the known logical relations between these notions.

The three notions of joint measurability provide operationally feasible ways of reconstructing the statistics of two observables $E_{1}, E_{2}$ from their joint observable. We went on to show that such operational reconstruction can even be achieved in cases where $E_{1}, E_{2}$ are not jointly measurable. We found important instances where from the statistics $p_{\rho}^{E}$ of a given observable $E$ one may, without the need of full state reconstruction, infer the statistics of another observable $p_{\rho}^{E_{1}}$ even though $\operatorname{ran}\left(E_{1}\right)$ is not contained in $\operatorname{ran}(E)$, and, a fortiori, there is no functional connection between $E_{1}$ and $E$. For example, the statistics of sharp position and momentum can be recovered from the statistics of a single phase space observable for a dense set of states. Hence, also in this case, the question (Q) has a positive answer without the observables involved being coexistent. "Measuring together" two observable that are not jointly measurable thus amounts to a common indirect measurement of the two observables, typically based on a measurement of a bi- or joint observable of unsharp versions or approximations of them.

For two observables that are coexistent or jointly measurable, there is an event, associated with an effect $E\left(Z_{X, Y}\right)$ from the joint or encompassing observable $E$, that represents the joint occurrence of two effects $E_{1}(X)$ and $E_{2}(Y)$. Such joint events do not exist for non-coexistent observables. This "deficiency" cannot be removed through a common indirect measurement. In this sense "measuring together" two observables in an indirect measuremet is a weaker notion than "measuring jointly". A more quantitative description of the idea of measuring two noncommuting sharp (hence non-coexistent) observables together "indirectly" has been obtained in investigations of recent years into a precise notion of approximate joint measurement. The examples given above, of joint measurements of unsharp versions of such sharp observables, can be considered as approximate joint measurements. The quality of the approximation is quantified by measures of inaccuracy, given by the distance between each one of the sharp observables from one of the marginals of the joint observable. Measurement uncertainty relations for such approximate joint measurements have been obtained, for example, in [38, 9, 28].

The existence of "joint events" for effects $E_{1}(X), E_{2}(Y)$ of two jointly measurable observables represented by effects $F(X \times Y)$ from a joint observable $F$ gives rise to an interpretation in terms of joint unsharp values that can be prepared by a suitable choice of measurement of $F$. An example of a weakly 
preparing measurement operation is given by the generalised Lüders operation associated with an effect $A$, defined as the map ([6])

$$
\rho \mapsto A^{1 / 2} \rho A^{1 / 2}
$$

We also noted that while joint measurability implies coexistence, it is not known whether there are coexistent observables that are not jointly measurable. Here we face the following two possibilities: It may be the case that coexistence is no more general than (one of) the three other notions of joint measurability; then the notion of coexistence adds no new possibilities. Alternatively, coexistence may turn out to be more general logically. There would thus be pairs of observables that are coexistent although they are not jointly measurable; but there seems to be no operational way of obtaining the probability distributions of the two observables in question from the encompassing observable. We conclude that the notion of coexistence has no added value over and above the three other notions of joint measurability. As far as the operational possibilities of joint measurements are concerned, one can safely use the term coexistence as a convenient synonym for joint measurability.

\section{REFERENCES}

[1] ALI, S. T.-PRUGOVEČKI, E.: Classical and quantum statistical mechanics in a common Liouville space, Phys. A 89 (1977), 501-521.

[2] BERG, C.-CHRISTENSEN, J.-RESSEL, P.: Harmonic Analysis on Semigroups, Springer, New York, 1984.

[3] BILlingSleY, P.: Probability and Measure (2nd ed.), John Wiley \& Sons, New York, 1986.

[4] BUSCH, P.: Unbestimmtheitsrelation und simultane Messungen in der Quantentheorie. PhD. Thesis, University of Cologne, Cologne, 1982

[English translation: Indeterminacy relations and simultaneous measurements in quantum theory, Internat. J. Theoret. Phys. 24 (1985), 63-92].

[5] BUSCH, P.: Unsharp reality and joint measurements for spin observables, Phys. Rev. D (3) 33 (1986), 2253-2261.

[6] BUSCH, P.: Some realizable joint measurements of complementary observables, Found. Phys. 27 (1987), 905-937.

[7] BUSCH, P.: On the sharpness and bias of quantum effects, Found. Phys. 39 (2009), 712-730. arXiv:0706.3532v2.

[8] BUSCH, P.-GRABOWSKI, M.-LAHTI, P. J.: Operational Quantum Physics. Lect. Notes Phys. Monogr., Springer, Berlin, 1995.

[9] BUSCH, P.-HEINONEN, T.-LAHTI, P.: Heisenberg's uncertainty principle, Phys. Rep. 452 (2007), 155-176.

[10] BUSCH, P.-KIUKAS, J.-LAHTI, P.: Measuring position and momentum together, Phys. Lett. A 372 (2008), 4379-4380.

[11] BUSCH, P.-SCHMIDT, H. J.: Coexistence of qubit effects, Quantum Inf. Process 9 (2010), 143-169. arXiv:0802.4167. 


\section{COEXISTENCE IN QUANTUM MECHANICS}

[12] CARMELI, C.-Heinonen, T.-TOIGO, A.: Position and momentum observables on $\mathbb{R}$ and $\mathbb{R}^{3}$, J. Math. Phys. 45 (2004), 2526-2539.

[13] DVUREČEnSKIJ, A.-LAHTI, P.-PULMANNOVÁ, S.-YLINEN, K.: Notes on coarse grainings and functions of observables, Rep. Math. Phys. 55 (2005), 241-248.

[14] HEINONEN, T. — LAHTI, P. - PELlONPÄ̈̈, J. P. — PUlMANNOVÁ, S. YLINEN, K.: The norm-1 property of a quantum observable, J. Math. Phys. 44 (2003), 1998-2008.

[15] HEINOSAARI, T.-REITZNER, D.-STANO, P.: Notes on joint measurability of quantum observables, Found. Phys. 38 (2008), 1133-1147.

[16] KIUKAS, J.-LAHTI, P.: A note on the measurement of phase space observables with an eight-port homodyne detector, J. Modern Opt. 55 (2008), 1891-1898.

[17] KIUKAS, J.-LAHTI, P.-PELLONPÄ̈̈, J. P.: A proof for the informational completeness for the rotated quadrature observables, J. Phys. A. Math. Theor. 41 (2008), 175206.

[18] KIUKAS, J.-LAHTI, P.-SCHULTZ, J.: Position and momentum tomography, Phys. Rev. A (3) 79 (2009), 052119.

[19] KIUKAS, J.-LAHTI, P.-YLINEN, K.: Semispectral measures as convolutions and their moment operators, J. Math. Phys. 49 (2008), 112103/6.

[20] KIUKAS, J.-WERNER, R.: Private communication, 2008.

[21] LAHTI, P.-PULMANNOVÁ, S.: Coexistent observables and effects in quantum mechanics, Rep. Math. Phys. 39 (1997), 339-351.

[22] LAHTI, P.-PULMANNOVÁ, S.: Coexistent vs. functional coexistence of quantum observables, Rep. Math. Phys. 47 (2001), 199-212.

[23] LAHTI, P.-PULMANNOVÁ, S.-YLINEN, K.: Coexistent observables and effects in convexity approach, J. Math. Phys. 39 (1998), 6364-6371.

[24] LAHTI, P.-YLINEN, K.: Dilations of positive operator measures and bimeasures related to quantum mechanics, Math. Slovaca 54 (2004), 169-189.

[25] LEONHARDT, U.-PAUL, H.-D'ARIANO, G. M.: Tomographic reconstruction of the density matrix via pattern functions, Phys. Rev. A (3) 52 (1995), 4899-4898.

[26] LUDWIG, G.: Foundations of Quantum Mechanics I, Springer, Berlin, 1983.

[27] MARTENS, H.-DE MUYNCK, W. M.: Nonideal quantum measurements, Found. Phys. 20 (1990), 255-281.

[28] MIYADERA, T.-IMAI, H.: Heisenberg's uncertainty principle for simultaneous measurement of positive-operator-valued measures, Phys. Rev. A (3) 78 (2008), 052119.

[29] MORELAND, T.-GUDDER, S.: Infima of Hilbert space effects, Linear Algebra Appl. 286 (1999), 1-17.

[30] PTÁK, P.-PULMANNOVÁ, S.: Orthomodular Structures as Quantum Logics. Kluwer Acad. Publ., Dordrecht, 1991.

[31] RAYMER, M. G.: Uncertainty principle for joint measurement of noncommuting variables, Amer. J. Phys. 62 (1994), 986-993.

[32] SIKORSKI, R.: Boolean Algebras, Springer, Berlin, 1964.

[33] SIMON, B.: The classical moment problem as a self-adjoint finite difference operator, Adv. Math. 137 (1998), 82-203.

[34] STANO, P.-REITZNER, D.-HEINOSAARI, T.: Coexistence of qubit effects, Phys. Rev. A (3) 78 (2008), 012315.

[35] TÖRM ̈̈, P.-STENHOLM, S.-JEX, I.: Measurement and preparation using two probe modes, Phys. Rev. A (3) 52 (1995), 4812-4822.

[36] VARADARAJAN, V. S.: Geometry of Quantum Theory, Springer, Berlin, 1985 (First edition by van Nostrand, Princeton, 1968, 1970). 


\section{PAUL BUSCH — JUKKA KIUKAS — PEKKA LAHTI}

[37] VON WEIZSÄCKER, C. F.: Quantum theory and space-time. In: Symposium on the Foundations of Modern Physics (P. Lahti, P. Mittelstaed, eds.), World Scientific Publishing Co., Singapore, 1985, pp. 223-237.

[38] WERNER, R.: The uncertainty relation for joint measurement of position and momentum, Quantum Inf. Comput. 4 (2004), 546-562.

[39] YU, S.-LIU, N. L.-LI, L.-OH, C. H.: Joint measurement of two unsharp observables of a qubit. arXiv:0805.1538 (2008).

Received 18. 5. 2009

Accepted 18. 5. 2009

\author{
* Department of Mathematics \\ University of York \\ York, YO10 5DD \\ UK \\ E-mail:pb516@york.ac.uk \\ ** Institut für Theoretische Physik \\ Universität Hannover \\ D-30167 Hannover \\ GERMANY \\ E-mail: Jukka.Kiukas@itp.uni-hannover.de \\ *** Turku Center for Quantum Physics \\ Department of Physics and Astronomy \\ University of Turku \\ FIN-20014 Turku \\ FINLAND \\ E-mail: pekka.lahti@utu.fi
}

